Acta Crystallographica Section A

Foundations and

Advances

ISSN 2053-2733

Received 19 February 2014

Accepted 11 July 2014

(C) 2014 International Union of Crystallography

\section{Use of bond-valence sums in modelling the diffuse scattering from PZN ( $\left.\mathrm{PbZn}_{1 / 3} \mathrm{Nb}_{2 / 3} \mathrm{O}_{3}\right)$}

\author{
R. E. Whitfield, ${ }^{\mathrm{a}, \mathrm{b} *}$ T. R. Welberry, ${ }^{\mathrm{a}}$ M. Paściak ${ }^{\mathrm{a}, \mathrm{c}}$ and D. J. Goossens ${ }^{\mathrm{a}}$ \\ ${ }^{a}$ Research School of Chemistry, Australian National University, Canberra, ACT 0200, Australia, \\ ${ }^{\mathbf{b}}$ Neutron Data Analysis and Visualization, Oak Ridge National Laboratory, Oak Ridge, TN 37831, \\ USA, and 'Institute of Physics, The Academy of Sciences of the Czech Republic, Prague, Czech \\ Republic. Correspondence e-mail: whitfieldre@ornl.gov
}

\begin{abstract}
This work extends previous efforts to model diffuse scattering from PZN $\left(\mathrm{PbZn}_{1 / 3} \mathrm{Nb}_{2 / 3} \mathrm{O}_{3}\right)$. Earlier work [Welberry et al. (2005). J. Appl. Cryst. 38, 639647; Welberry et al. (2006). Phys. Rev. B, 74, 224108] is highly prescriptive, using Monte Carlo simulation with very artificial potentials to induce short-rangeorder structures which were deduced as necessary from inspection of the data. While this gives valid results for the nature of the local structure, it does not strongly relate these structures to underlying crystal chemistry. In that work, the idea of the bond-valence sum was used as a guide to the expected behaviour of the atoms. This paper extends the use of the bond-valence sum from a qualitative guide to becoming a key aspect of the potential experienced by the atoms, through the idea of the global instability index, whose square has been shown to be proportional to the density functional theory energy of some systems when close to the minimum energy configuration.
\end{abstract}

\section{Introduction}

There have been a number of high-quality powder diffraction studies of $\mathrm{PZN}\left(\mathrm{PbZn}_{1 / 3} \mathrm{Nb}_{2 / 3} \mathrm{O}_{3}\right)$ (Forrester et al., 2006; Terado et al., 2006). However, the considerable detail available in the single-crystal diffuse scattering (SCDS), and its likely relation to the physical properties of the material, suggest very strongly that it is necessary to go beyond the average structure (Welberry et al., 2005; Xu et al., 2008; Burkovsky, Bronwald et al., 2012; Burkovsky, Filimonov et al., 2012; Paściak et al., 2012; Paściak \& Welberry, 2011).

Studies of diffuse scattering from functional oxide materials such as PZN and similar (Paściak et al., 2007; Welberry et al., $2005,2006)$ reveal considerable detail about the polar nanodomain structure considered to be so important in determining the behaviour of these technologically significant materials (Xu et al., 2008). [Although that idea can be challenged (Bosak et al., 2012; Hlinka, 2012).]

Use of Monte Carlo (MC) computer simulations of a model structure has become a powerful and well accepted technique for aiding the interpretation and analysis of diffuse scattering patterns (Welberry \& Butler, 1994; Weber et al., 2001; Weber \& Bürgi, 2002; Welberry, 2004; Paściak et al., 2010). However, there are inevitable compromises in the modelling. Model systems large enough to model the population of local configurations are generally found to be on the order of $48 \times 48 \times 48$ unit cells, a size that precludes detailed modelling of the potentials, for example using density functional theory (DFT).
However, it has been shown that, in inorganic systems such as oxides, the bond-valence-sum (BVS) approach (Brese \& O'Keeffe, 1991; Brown, 2009; Adams, 2001) leads to a measure of system structural stability, the so-called 'Global instability index', $R_{1}$ or Gii, whose square often scales with the energy when close to the minimum (Perez-Mato et al., 2008; Etxebarria et al., 2005). However, such a relationship is likely only reliable for 'small distortions that do not change the basic topology of the structure' (Etxebarria et al., 2005). In the modelling of disorder, where the average structure is taken as the starting point and the disorder can be thought of as structural modulations (compositional or displacive) that modify that average on the local scale, this should be a well obeyed assumption. Reverse Monte Carlo (RMC) modelling of disordered systems has made use of bondvalence considerations to provide constraints (Norberg et al., 2009, 2011).

Gii is the root-mean-square over all atoms of the difference between the apparent valence $V_{i}$ (which comes from a sum over the valences of the bonds to which the atom contributes; this is the BVS) and the nominal valence, $V_{i}^{0}$. The apparent valence is calculated from the given structure in the usual way using $R_{\mathrm{O}-\mathrm{Pb}}=2.112, R_{\mathrm{O}-\mathrm{Zn}}=$ $1.704, R_{\mathrm{O}-\mathrm{Nb}}=1.911$ and $R_{\mathrm{O}-\mathrm{O}}=1.48$ (Brese \& O'Keeffe, 1991),

$$
V_{i}=\sum_{j} v_{i j}
$$

where 


$$
v_{i j}=\exp \left(\frac{d_{i j}-R_{i j}}{b}\right),
$$

where the observed atomic separation is $d_{i j}$, the bond-valence parameter is $R_{i j}$ and $b$ is a constant, often given as 0.37 but perhaps less universal than was once thought (see, for example, Mills et al., 2009; Sidey, 2010).

Then

$$
\mathrm{Gii}^{2}=\frac{1}{N} \sum_{i=1}^{N}\left(V_{i}-V_{i}^{0}\right)^{2} .
$$

Unlike detailed $a b$ initio methods, the BVS are quick to calculate.

The work presented here explores the ability of the $\mathrm{Gii}^{2}$ to act as a valid system 'energy' in the context of MC simulation.

However, the BVS is a scalar quantity and comes from a scalar calculation of atomic distances. Hence it does not work well in deducing rigid-body motions (such as octahedral rotation). [Some work has been undertaken on exploring vectorial sum rules (Harvey et al., 2006).] Further, the interesting behaviour of the $\mathrm{Pb}^{2+}$ atoms in materials like PZN and
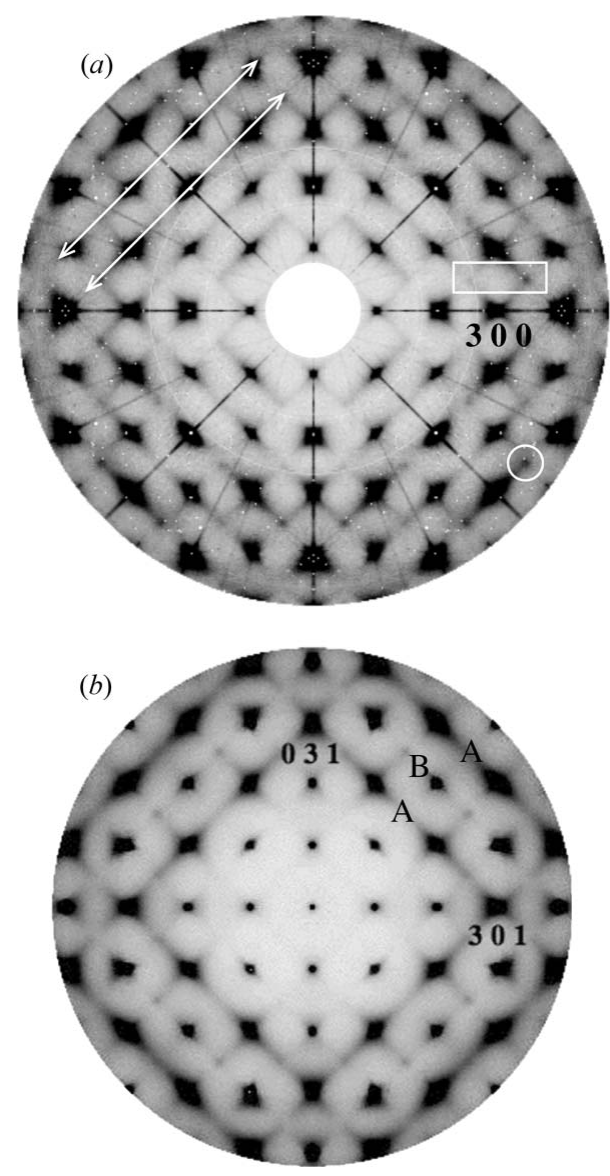

Figure 1

Sections of neutron diffuse scattering from PZN, measured at room temperature. (a) $h k 0$ measured using Wombat at OPAL and (b) $h k 1$ measured using SXD at ISIS. (a) shows some signs of radial streaking and concentric rings (instrumental effects) plus some arcs due to the application of symmetry to the data. However, the diffuse streaks (examples are noted by white arrows) are clear and sharp. On this scale strong scattering is darker.
PMN $\left(\mathrm{PbMg}_{1 / 3} \mathrm{Nb}_{2 / 3} \mathrm{O}_{3}\right)$ is thought to be strongly connected to the polarity of the ion due to its lone pair; such an effect is also not well modelled by BVS, since the natural polarity of the atom will influence the mechanism by which the atom optimizes its valence; whether, for example, a relatively isotropic contraction or expansion of the oxygen environment takes place, or whether the atom shifts off-centre to satisfy its bonding requirements. Plainly, this will be crucial in accurately modelling the atom's behaviour. Therefore, the model presented here incorporates aspects of the prescriptive and somewhat artificial approach used previously (for example in Welberry et al., 2005), along with a more general approach using the BVS (through $\mathrm{Gii}^{2}$ ) as a substitute for detailed potentials such as those from DFT (Perez-Mato et al., 2008; Etxebarria et al., 2005).

\section{Experimental method}

Diffuse scattering data from PZN have been collected using the Wombat high-intensity diffractometer at the OPAL reactor at the Bragg Institute, Australian Nuclear Science and Technology Organization (ANSTO) (Studer et al., 2006; Whitfield, Goossens \& Studer, 2012; Whitfield, Goossens, Studer \& Forrester, 2012) and the 11-ID-B beamline at the Advanced Photon Source, as well as previously at the ISIS neutron source (Welberry et al., 2005).

In all cases, a three-dimensional volume of scattering was collected. The data were then reconstructed and corrected to present an undistorted slice of reciprocal space, or number of slices, for example in Fig. 1.

\section{Modelling approach}

Fig. 1 shows the $h k 0$ section of reciprocal space measured at $300 \mathrm{~K}$ at ANSTO and the $h k 1$ layer of diffuse scattering measured in the ferroelectric phase at room temperature using SXD at ISIS.

Circled on Fig. 1(a) is an example of a quite strong diffuse peak at $h k l \pm \frac{1}{2} \frac{1}{2} 0$, the result of intersections of many $\langle 110\rangle$ type streaks (two such streaks, in fact rods of scattering, are noted in Fig. 1a). Note the strong size effect, indicated by the difference in intensity between the two such diffuse peaks enclosed in a rectangular box in Fig. 1(a), and the quite asymmetric regions of diffuse scattering found around most Bragg peaks - the 300 peak is noted for scale, but the asymmetry is more pronounced around the next peak out - the 400 . The size-effect diffuse scattering is related to the local relaxations of neighbouring atom positions related to the different atom sizes. In the case of this system, the effective 'size' of the $\mathrm{Pb}$ atoms is dependent on the respective displacement of the neighbouring atoms.

A further key feature is the variation in intensity of the diffuse rods. In $h k 0$ all the rods are of similar strength. They do show modulation in intensity as a function of position along the rod. In the $h k 1$ section every second rod is substantially more intense relative to the others. Rods denoted ' $\mathrm{A}$ ' are stronger than the intervening rod labelled ' $\mathrm{B}$ '. 
Table 1

ADPs used in the MC simulations, and the magnitude of the $\langle 110\rangle \mathrm{Pb}$ shift, $\delta_{\mathrm{Pb}}$.

ADPs are in $10^{-2} \AA^{2}, \delta$ is in $\AA$.

\begin{tabular}{llll}
\hline Atom ADP & $\begin{array}{l}\text { Terado } \text { et al. (2006) } \\
650 \mathrm{~K}, \operatorname{Pm} \overline{3} m\end{array}$ & $\begin{array}{l}\text { Forrester } \text { et al. (2006) } \\
295 \mathrm{~K}, R 3 m\end{array}$ & This work \\
\hline$U_{\mathrm{Pb}}$ & $2.37(8)$ & & 2.4 \\
$U_{\mathrm{Pb} 11}$ & & $4.95(8)$ & \\
$U_{\mathrm{Pb} 12}$ & & $-1.20(10)$ & \\
$\delta_{\mathrm{Pb}}$ & $0.299(2)$ & & 0.3 \\
$U_{\mathrm{O} 11}$ & $1.53(16)$ & $2.30(1)$ & 1.5 \\
$U_{\mathrm{O} 22}$ & $3.20(25)$ & $2.88(7)$ & 3.2 \\
$U_{\mathrm{O} 12}$ & & $-0.13(9)$ & \\
$U_{\mathrm{O} 23}$ & & $-0.50(10)$ & 1.5 \\
$U_{\mathrm{Nb} / \mathrm{Zn}}$ & $1.46(8)$ & $1.67(5)$ & \\
\hline
\end{tabular}

The diffuse scattering from PZN was modelled rather artificially through interacting random variables (Welberry et al., 2005). Later work has tried to improve on this through a more atomistic simulation (Welberry et al., 2006); but even in that case the non- $\mathrm{Pb}$ atoms were placed deterministically into the simulation, based on the behaviour of the $\mathrm{Pb}$ atoms. Here, the BVS and $\mathrm{Gii}^{2}$ are used to determine these atomic positions. And to further refine the approach and ensure that the resulting model would be consistent with the published conventional diffraction studies, other variations from the process laid out in the earlier works were implemented.

In particular, the atoms in the model presented here were all constrained to have displacive distributions (atomic displacement parameters, ADPs) consistent with previous results (Forrester et al., 2006; Terado et al., 2006). The data were collected at room temperature, and so on first inspection the correct structural model to use is that published by Forrester et al. (2006). However, while that model is perfectly

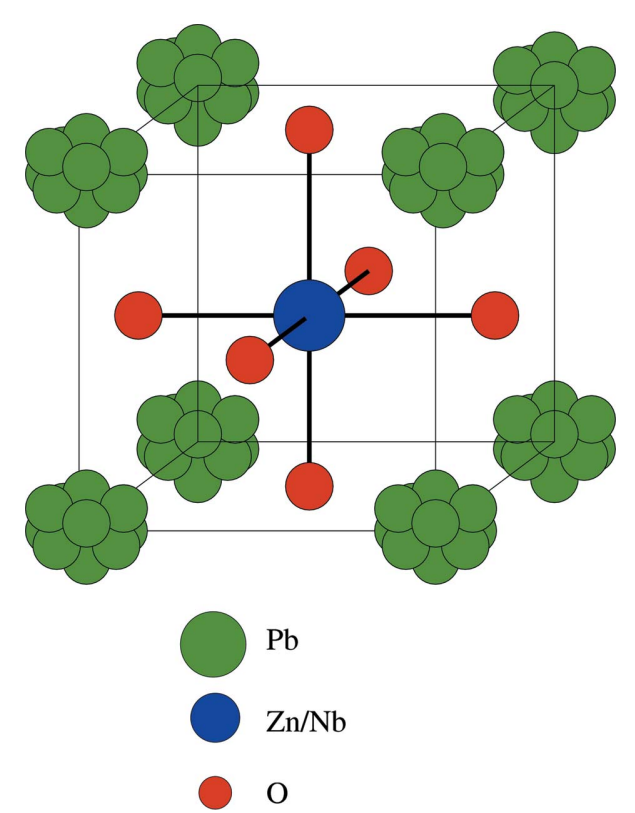

Figure 2

The $\mathrm{Pb}$ displacement model in the cubic phase of PZN-PT $\left[(1-x) \mathrm{Pb}\left(\mathrm{Zn}_{1 / 3} \mathrm{Nb}_{2 / 3}\right) \mathrm{O}_{3-x} \mathrm{PbTiO}_{3}\right]$ where $0<x<0.15$ (Terado et al., 2006). reasonable in terms of the ability of the powder diffraction data to discriminate against the sort of disorder present in PZN, the very existence of the structured diffuse scattering at room temperature means that there must be a disordered distribution of $\mathrm{Pb}$ ions such that the displacements can then be correlated to give the observed diffuse scattering. Terado et al. (2006) looked at the structure at high temperature, in the cubic phase, and found that there is a disordered distribution of $\mathrm{Pb}$ atoms, with the $\mathrm{Pb}$ atoms equally distributed across the $12\langle 110\rangle$ displacements. Hence, the total $\mathrm{Pb}$ distribution can be obtained by putting $\frac{1}{12}$ of a $\mathrm{Pb}$ atom at each of the [110]-type displaced positions and then applying to that fractional atom an isotropic ADP. There is no consensus on the actual displacement direction of the $\mathrm{Pb}$ atoms (Welberry et al., 2006), such as in Jeong \& Lee (2006) who determined the shift along $\langle 111\rangle$ directions, while Dmowski et al. (2000) found the $\langle 100\rangle$ direction was most likely; the $\langle 110\rangle$ direction was used as previous similar modelling showed this to be best (Welberry et al., 2005).

To determine a set of ADPs to be used in modelling the SCDS, the results of both these works were combined; both give similar ADPs for non- $\mathrm{Pb}$ atoms and, indeed, when the ADP for the $\mathrm{Pb}$ atom in the model from Terado et al. (2006) is calculated as if it were a single ordered site, the result is very close to that obtained in Forrester et al. (2006). Hence the procedure was to generate random atomic distributions in accordance with the published ADPs, and then perform any MC simulation by swapping displacements amongst equivalent atoms, thereby maintaining the overall distribution and so maintaining the ADPs and demonstrating that the local ordering can be modelled while maintaining the accepted average structure.

The second major departure from the previous studies was the use of $\mathrm{Gii}^{2}$ as an energy term in an MC simulation to establish the positions of the non- $\mathrm{Pb}$ atoms once the $\mathrm{Pb}$ positions had been determined. As noted above, the bondvalence approach was not expected to work well in determining the correlations in the $\mathrm{Pb}$ displacements (and tests showed it did not) because of the polar nature of the $\mathrm{Pb}^{2+}$ ion. Hence, $\mathrm{Gii}^{2}$ minimization was used to position $\mathrm{O}, \mathrm{Nb}$ and $\mathrm{Zn}$ within a $\mathrm{Pb}$ framework fixed by earlier stages of the simulation.

In outline, the simulation was as follows:

(a) Establish an initial configuration with $\mathrm{Nb}$ and $\mathrm{Zn}$ atom types randomly distributed on the $B$ sites, with ADPs taken from the literature (see Table 1); with $\mathrm{O}$ distributed according to the literature and with $\mathrm{Pb}$ ions randomly distributed across the $12\langle 110\rangle$-type displaced positions, with a displacement magnitude of $\delta_{\mathrm{Pb}}$. The ADPs are applied to the atom sites by randomly displacing the atom using a Gaussian distribution, with the ADPs being conserved across the model.

(b) Following previous work (Welberry et al., 2006), (i) use an Ising energy to induce correlation in the $B$-site occupancy, and (ii) also induce the polar nanodomain structure in the $\mathrm{Pb}$ displacement distribution.

(c) Add an isotropic ADP to each of the $12 \mathrm{~Pb}$ positions, $U_{\mathrm{Pb}}$, see Table 1 , in the form of additional random atom 
displacements on top of the 'large' $\langle 110\rangle$-type displacement. This produces the 'bunch of grapes' displacement distribution, Fig. 2, as found in powder diffraction work (Terado et al., 2006).

(d) In contrast to previous work, a swapping algorithm instead of displacive algorithm is used to apply the size effect to the $\mathrm{Pb}$ distribution. The magnitude and direction of the effect are taken from earlier work (Welberry et al., 2005, 2006) but are manifested through swapping the isotropic ADP component [added in step $(c)$ ] of the atomic coordinates, swapping between $\mathrm{Pb}$ ions with the same type of $\langle 110\rangle$ displacement, allowing the initial ADPs introduced to remain.

(e) Unlike previous simulations the displacement of the other atoms is determined by swapping the ADP displacements of $\mathrm{Zn}$ with $\mathrm{Zn}, \mathrm{Nb}$ with $\mathrm{Nb}$, and $\mathrm{O}_{i}$ with $\mathrm{O}_{i}(i=1,2,3)$. Allowing the swapping of atomic displacement between $\mathrm{Zn}$ and $\mathrm{Nb}$ atoms is explored in $\$ 5.2$.

\section{Results and discussion}

\subsection{Ising model $-B$-site ordering}

In PZN the $B$ site has a mixture of $\mathrm{Zn}$ and $\mathrm{Nb}$ with an occupancy fraction of $\frac{1}{3}$ and $\frac{2}{3}$, respectively. The $\mathrm{Zn}$ and $\mathrm{Nb}$ atoms appear to try to order in an alternating pattern with negative correlation, but are unable to do so completely, so producing a disordered but correlated chemical ordering on this site (Welberry et al., 2005). This manifests in broad $\frac{1}{2} \frac{1}{2} \frac{1}{-}^{-}$ type superlattice spots in the diffraction pattern. A plot of one layer from the model crystal, Fig. 3, shows the $\mathrm{Zn}$ (blue) and

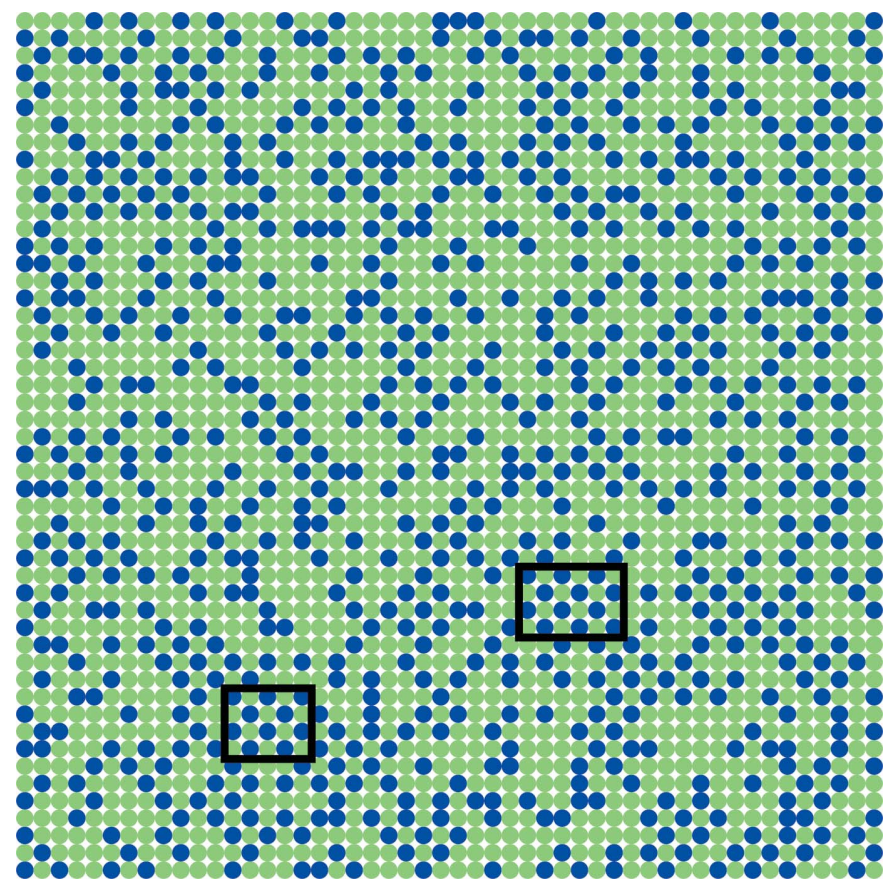

Figure 3

An $a, b$ layer from the model crystal showing the $\mathrm{Zn}$ (blue) and $\mathrm{Nb}$ (green) atoms. The atoms can be seen to try to arrange with alternating types, with negative correlation. Two areas of complete alternating ordering are highlighted.
$\mathrm{Nb}$ (green) atoms. The atoms can be seen to try to form in a alternating pattern.

\subsection{Planar nanodomain formation}

The planar nanodomain formation was implemented with the same artificial energy term as outlined in detail in previous work (Welberry et al., 2005, 2006).

The initial setup of the model crystal involved the random positioning of each of the $\mathrm{Pb}$ atoms in one of the $12\langle 110\rangle$ directions. The atomic displacement had a magnitude of $\delta_{\mathrm{Pb}}$ as seen in Table 1. A depiction of the arrangement of the initial $\mathrm{Pb}$ displacements is given in Fig. 4. The swapping MC method was used where the $\mathrm{Pb}$-atom $\langle 110\rangle$ displacements are swapped. The resulting formation of domains is shown in Fig. 5 and domains range in size up to about $40 \AA$ or ten unit cells.

The resulting diffuse scattering calculated from this model is shown in Fig. 6. Other than the diffuse peaks occurring at the $\frac{1}{2} \frac{1}{2}$-type positions, only the $\mathrm{Pb}$ atoms contribute to the diffuse scattering because thus far the other atoms all sit on their

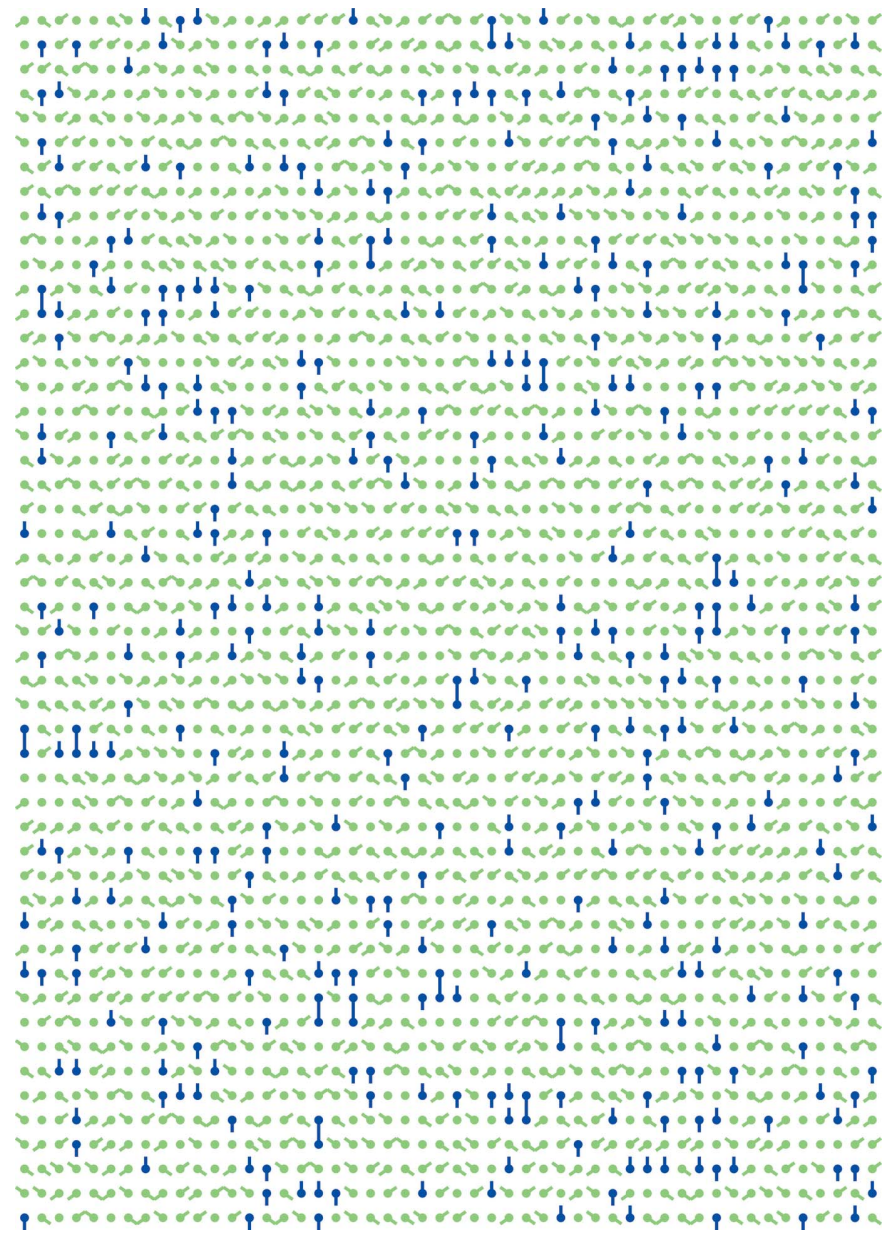

Figure 4

A two-dimensional real-space plot with a normal [011] of one layer in the model crystal showing the initial arrangement of $\mathrm{Pb}$ displacements, not to scale, before the formation of the planar nanodomains. Highlighted with blue are the $\mathrm{Pb}$ displacements which are in the plane of the figure, [011] and $[0 \overline{11}]$. The same crystal model layer is shown after the domain formation in Fig. 5. 
average positions and will only contribute to Bragg scattering. The diffuse rods of scattering in the $\langle 110\rangle$ directions can be seen and are highlighted in Fig. 6(a). The intensity and width of the diffuse rods are mostly consistent along their lengths, which is not what is seen in the data, and the intensity is also similar between the different $h k 0$ and $h k 1$ planes.

The intersections of the diffuse rods with the $h k \frac{1}{2}$ plane can be seen in Figs. 6(c) (an example boxed in green) and 6(d) and produce broader diffuse scattering than the $B$-site ordering diffuse scattering peaks.

\subsection{Size effect}

It is clear that size effect, the interaction of occupancy and displacement, is important in modelling the diffuse scattering (Welberry et al., 2006). In this case, 'occupancy' refers to which of the $12\langle 110\rangle$-type positions the atom occupies. While here the same energy term for the size effect is used as previously, the implementation is different. Instead of small random

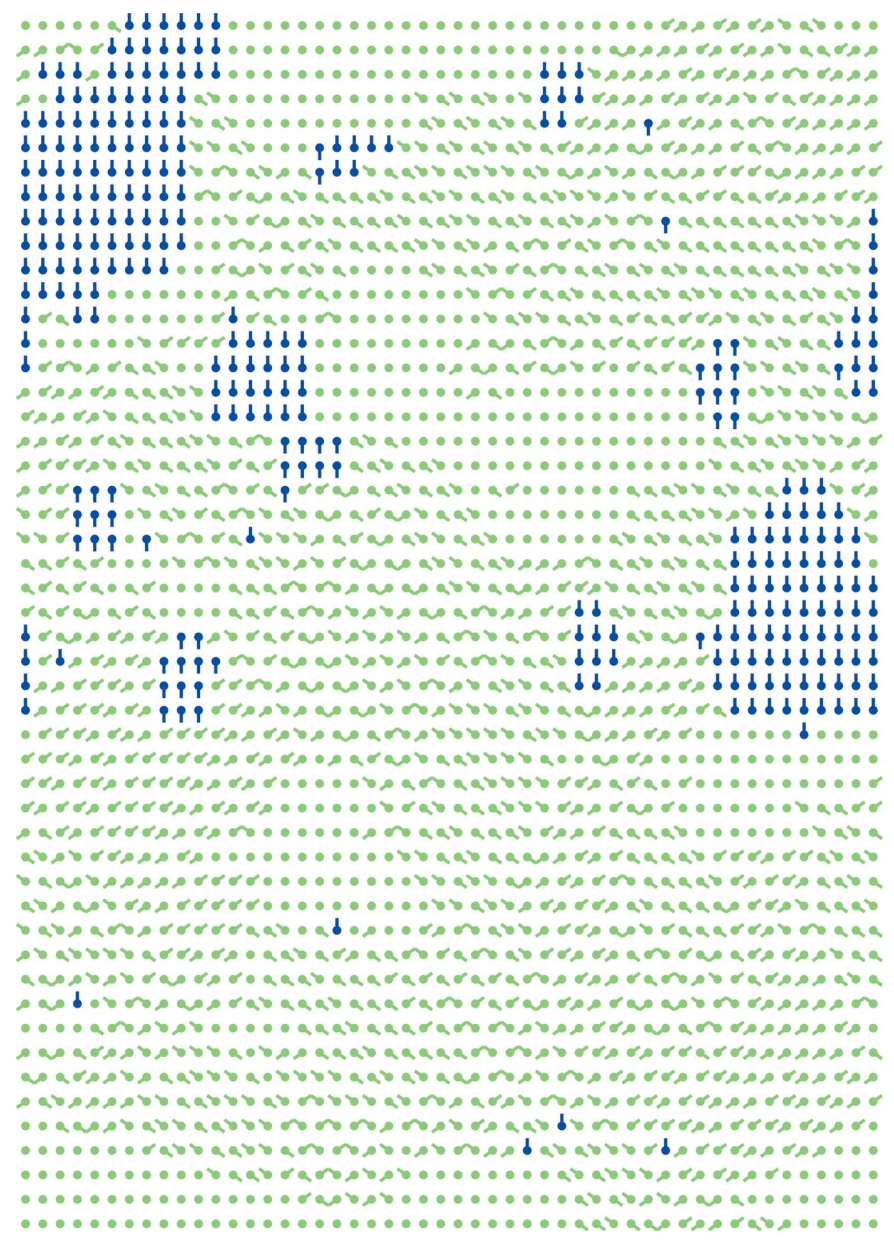

\section{Figure 5}

A two-dimensional real-space plot with a normal [011] of one layer in the model crystal showing the arrangement of $\mathrm{Pb}$ displacement after the MC simulation and the formation of the planar nanodomains. The initial distribution of $\mathrm{Pb}$ displacements before the $\mathrm{MC}$ simulation is shown in Fig. 4. Highlighted with blue are the $\mathrm{Pb}$ displacements which are in the plane of the figure, [011] and [011]. The domains can be seen to vary in sizes up to around ten unit cells or about $40 \AA$. atomic displacements being added to atom coordinates, first a random displacement is added to each of the $\mathrm{Pb}$-atom sites so that, averaging over the complete model, ADPs are reproduced as listed in Table 1 . Then during the MC simulation, only the atom displacements that were added on top of the $\delta_{\mathrm{Pb}}$ displacement are swapped between $\mathrm{Pb}$ atoms of like $\langle 110\rangle$ displacement, conserving the planar domain formation and the overall thermal disorder of the atom site.
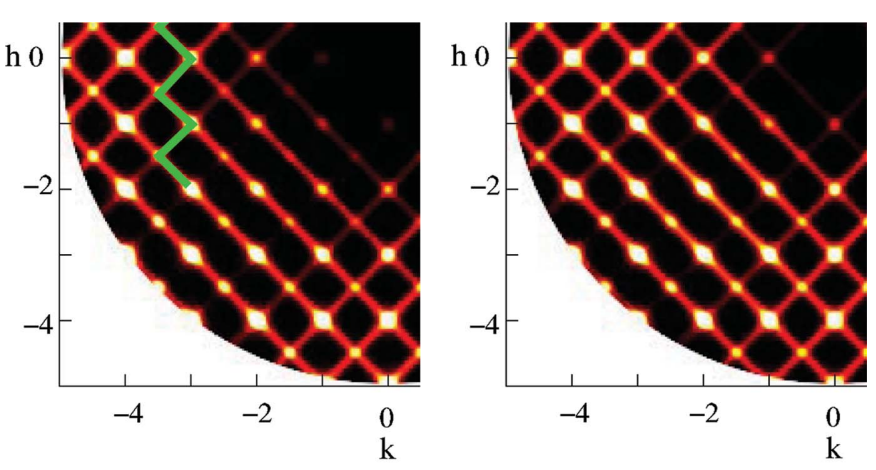

(a) $h k 0$ Neutron

(b) $h k 0 \mathrm{X}$-ray
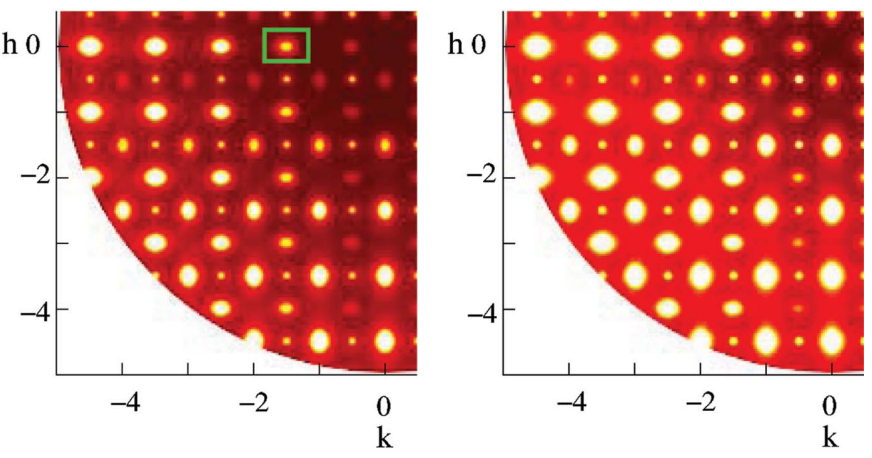

(c) $h k^{1 / 2}$ Neutron

(d) $h k^{1 / 2} \mathrm{X}$-ray
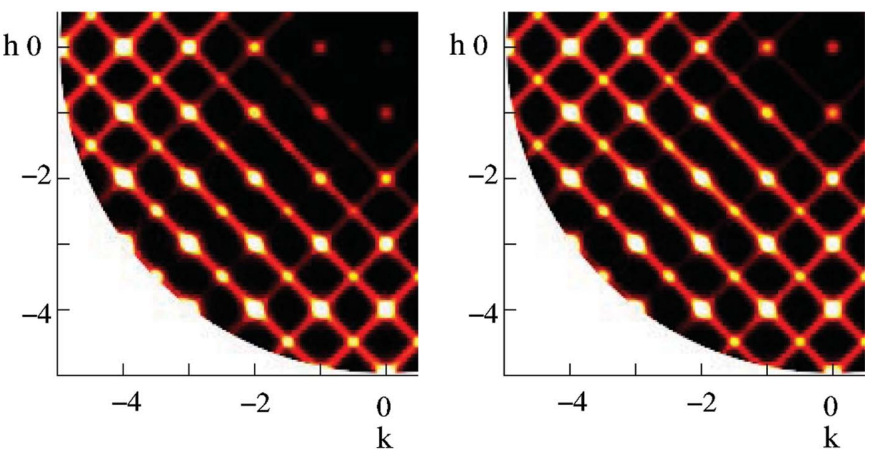

(e) $h k 1$ Neutron

(f) $h k 1$ X-ray

\section{Figure 6}

The formation of the planar domains in the crystal model produces the diffuse rods as seen in the calculated diffraction patterns in the $\langle 110\rangle$ directions away from the Bragg peaks. The diffuse scattering from the model crystal is shown for both neutrons and X-rays for the planes $h k 0$, $h k \frac{1}{2}$ and $h k 1$. The $h k \frac{1}{2}$ plane is on a different intensity scale compared to the other planes. The diffuse rods are highlighted in $(a)$ with the zigzag line. A peak in the $h k \frac{1}{2}$, formed by intersection of a rod of scattering with this plane, is boxed in $(c)$. On these figures black is weak scattering while white is stronger. 
The result that the size effect has on the diffraction pattern of the model can be observed in Fig. 7. When compared to the previous scattering, Fig. 6 , the redistribution of intensity is apparent, with a reduction in the symmetry of the scattering on the Bragg positions when comparing the high- $q$ and low- $q$ sides, as seen in the data (Fig. 1).

The intersection of the diffuse rods with the $h k \frac{1}{2}$ plane shows that the rods decrease in intensity and increase in width. This is somewhat expected as the $\mathrm{Pb}$ displacements do become more correlated but the planar domains, particularly the
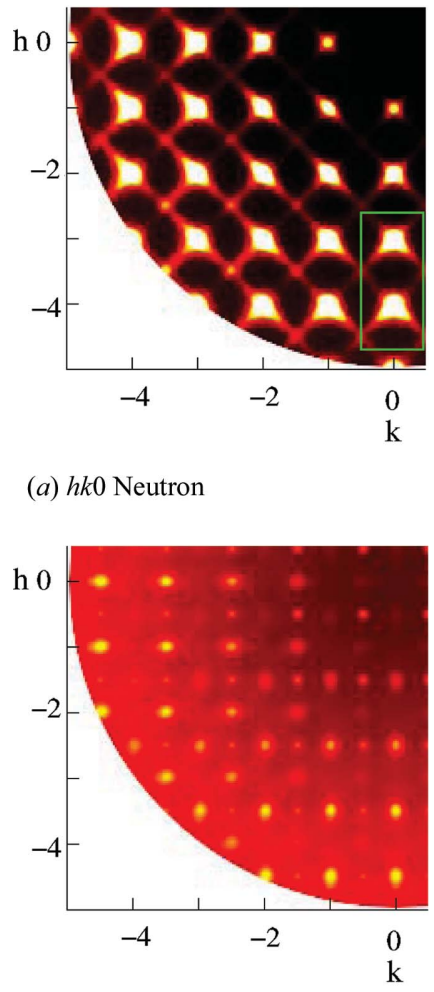

(c) $h k^{1 / 2}$ Neutron

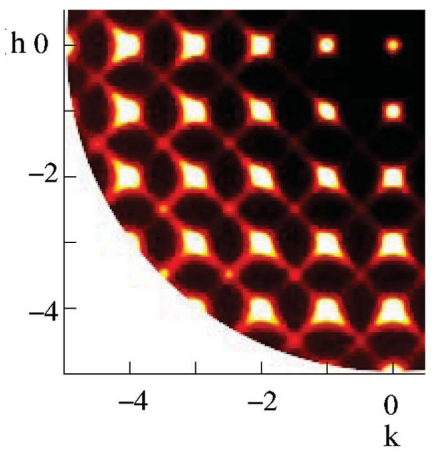

(e) $h k 1$ Neutron (a) $h k 0$ Neutron

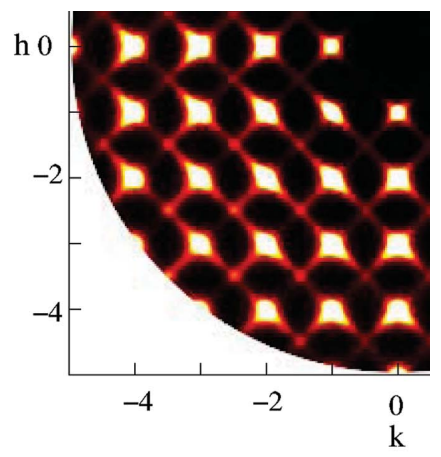

(b) $h k 0 \mathrm{X}$-ray

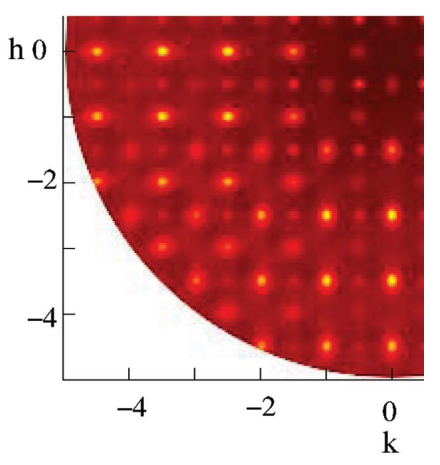

(d) $h k^{1 / 2} \mathrm{X}$-ray

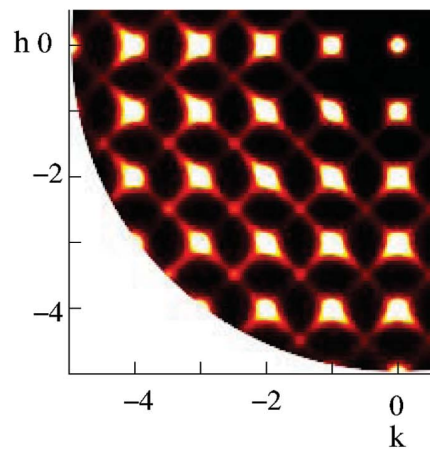

(f) $h k 1$ X-ray

\section{Figure 7}

The calculated diffuse scattering from the model crystal for different reciprocal planes, $h k 0, h k \frac{1}{2}$ and $h k 1$, comparing both neutrons and X-rays after the size effect has been applied. The size-effect MC simulation creates the structured diffuse scattering seen around the Bragg peaks, some examples boxed in $(a)$. Diffuse scattering without size effect can be seen in Fig. 6. There is little difference between the neutron and X-ray patterns at this point, as most of the scattering is due to only the $\mathrm{Pb}$ atoms.
Table 2

Displacement correlation $c_{\mathrm{Pb}-\mathrm{Pb}}^{x}$ for the $a$-axis component of the displacements at neighbouring $\mathrm{Pb}$ sites for each stage in the modelling process.

The different displacement component correlations, $c_{\mathrm{Pb}-\mathrm{Pb}}^{x}, c_{\mathrm{Pb}-\mathrm{Pb}}^{y}$ and $c_{\mathrm{Pb}-\mathrm{Pb}}^{z}$, are consistent for $\mathrm{Pb}$.

\begin{tabular}{ll}
\hline Simulation stage & $\begin{array}{l}\mathrm{Pb}-\mathrm{Pb} x \text { displacement } \\
\text { correlation }\end{array}$ \\
\hline Random $\mathrm{Pb}\langle 110\rangle$ displacement & 0.000 \\
Planar domain formation & 0.262 \\
Thermal parameter applied & 0.142 \\
Size-effect applied & 0.562 \\
\hline
\end{tabular}

domain edges, become less well defined due to the relaxation of the atomic displacements.

The displacement correlation coefficients, $c_{\mathrm{Pb}-\mathrm{Pb}}^{x}$ as defined by equation (4), calculated for neighbouring $i$ and $j$ atoms,

$$
c_{i j}^{x}=\frac{\left\langle x_{i} x_{j}\right\rangle}{\left(\left\langle x_{i}^{2}\right\rangle\left\langle x_{j}^{2}\right\rangle\right)^{1 / 2}}
$$

where $x_{i}$ is the displacement along the $a$ axis of the $i$ th $\mathrm{Pb}$ ion, can be calculated for different steps in the MC simulation process. This is shown in Table 2, which illustrates the effect each step has on the Pb-atom local structure. Initially it starts with a random distribution of $\mathrm{Pb}$ displacements with approximately zero correlation and after the domain formation the correlation is increased to about 0.26 . The addition of the thermal parameters decreases the correlations due to the random displacement of the atoms. When the size-effect simulation is applied, the $\mathrm{Pb}$ displacements become highly correlated with nearly a 0.56 correlation coefficient.

A plot showing the change of the distribution of the $\mathrm{Pb}$ atom distances before and after the size-effect calculation is shown in Fig. 8, which gives more insight into the local

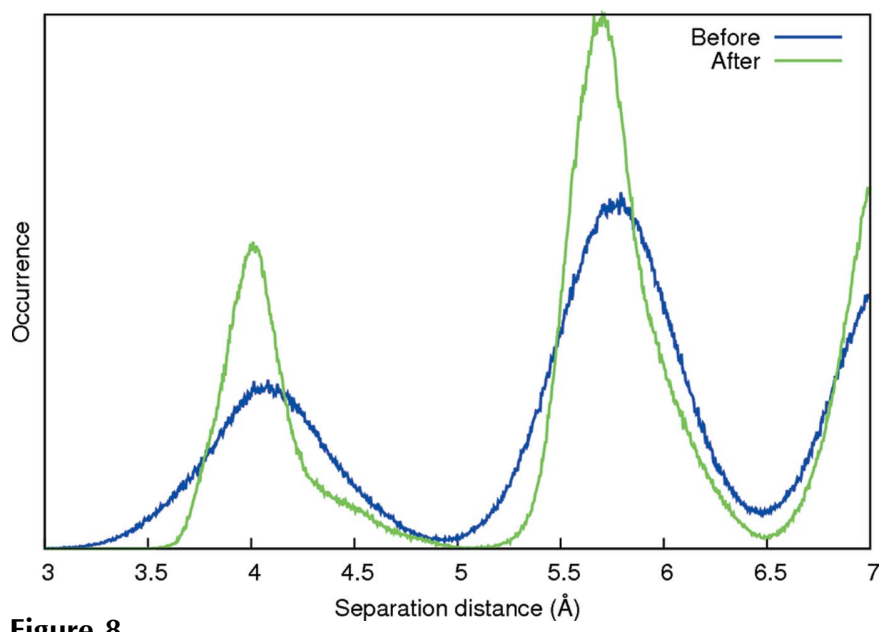

Figure 8

The distribution of separation distances between $\mathrm{Pb}$ atoms in neighbouring cells before the size effect is applied and after. The change in the distribution is due to the increase of correlations of the $\mathrm{Pb}$-atom displacements. 


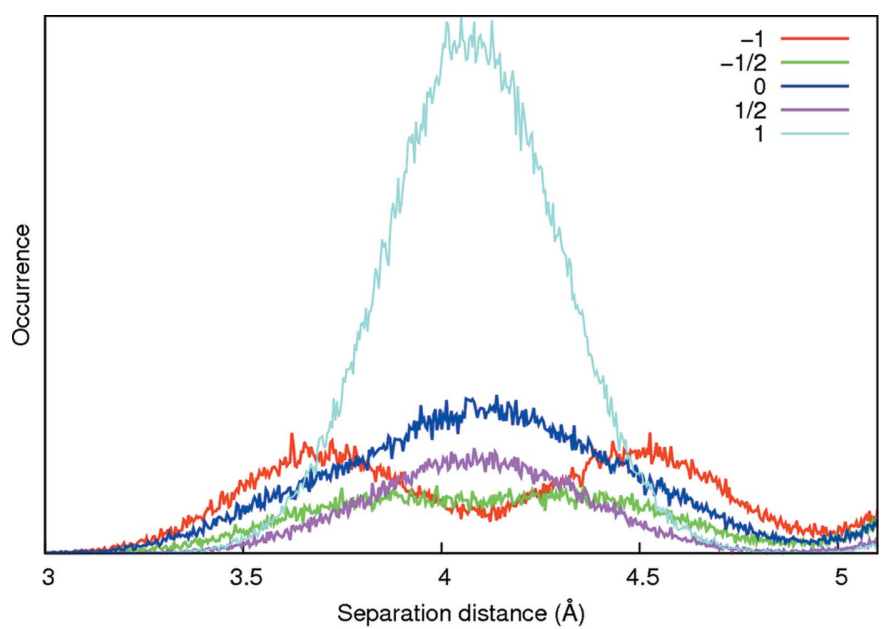

(a)

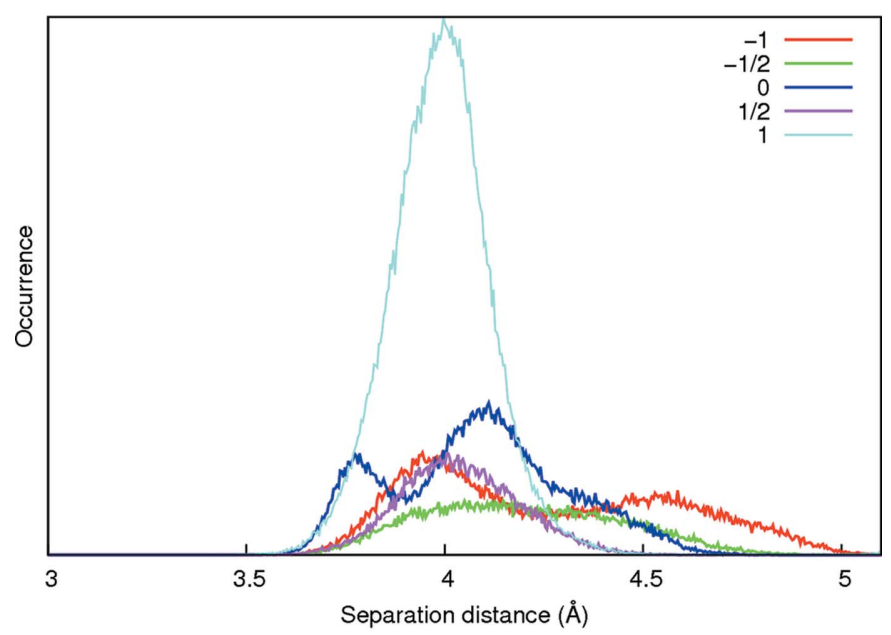

(b)

Figure 9

Plot of the distribution of $\mathrm{Pb}$ separation distances, depending on $\chi_{i, j}$, as calculated from $\chi_{i, j}=\hat{\delta}^{i} \cdot \hat{\delta}^{j}$, for before and after the size effect is applied. A $\chi=1$ occurs when the neighbouring $\mathrm{Pb}$ displacements are parallel and in the same direction. $\chi=-1$ when they are in the opposite directions. The neighbouring $\mathrm{Pb}$ atoms with $\chi=1$ are the most common, as this occurs for the formation of the planar nanodomains.

structure of the $\mathrm{Pb}$ atoms than the correlation coefficient. Initially the distribution of distances appears to be symmetrical and Gaussian, but after the size effect the shape changes with a shift of the peak to a shorter distance. The distribution also becomes sharper with the increase of the displacement correlations.

The asymmetric shape of the nearest-neighbour $\mathrm{Pb}-\mathrm{Pb}$ separation is further explored in Fig. 9, where the peak is separated into components. Each component is for atom pairs with different relative displacement directions, $\delta^{i}$ and $\delta^{j}$. If these are normalized, their dot product, $\chi_{i, j}=\hat{\delta}^{i} \cdot \hat{\delta}^{j}$, can be used to classify interatomic $\mathrm{Pb}-\mathrm{Pb}$ distances. If $\chi=1$ the neighbouring $\mathrm{Pb}$ displacements are parallel, if 0 then orthogonal and if -1 antiparallel, for example. The size effect is such that $\chi$ values of $\frac{1}{2}$ or 1 cause a decrease in the separation distance, while $\chi$ values of $-\frac{1}{2}$ or -1 cause an increase in the separation distance. This results in an asymmetric distribution of $\mathrm{Pb}-\mathrm{Pb}$ distances.

\section{Bond-valence sum}

To this point, the changes to the modelling have been essentially to use swapping algorithms to ensure that the average structure is explicitly maintained. Now, $\mathrm{Gii}^{2}$ [equation (3)] is used as an MC energy term to determine the non-Pb-atom positions in a more physically realistic way. $\mathrm{Pb}$ atoms are held fixed. The non-Pb-atom displacements are swapped between equivalent sites, conserving their overall thermal displacement parameters and occupancies. The swapping of the atoms reduces $\mathrm{Gii}^{2}$ until it reaches an equilibrium value.

\subsection{Results of the BVS Monte Carlo}

In this model, the displacements of $\mathrm{Nb}$ ions were swapped with other $\mathrm{Nb}, \mathrm{Zn}$ with other $\mathrm{Zn}$, and $\mathrm{O}_{i}$ with $\mathrm{O}_{i}$ where $i=1,2,3$ are the three $\mathrm{O}$ atoms in the unit cell. The diffuse scattering calculated from this model is shown in Fig. 10. The changes in the diffuse scattering compared to the previous calculation, Fig. 7, are subtle, so the differences between the scattering from the previous size-effect simulation and the BVS model are shown in Fig. 11, calculated by pixel-by-pixel subtraction of the images. The difference plots for X-rays and neutrons are on different intensity scales, so are not directly comparable. The greatest change can be seen in the neutron
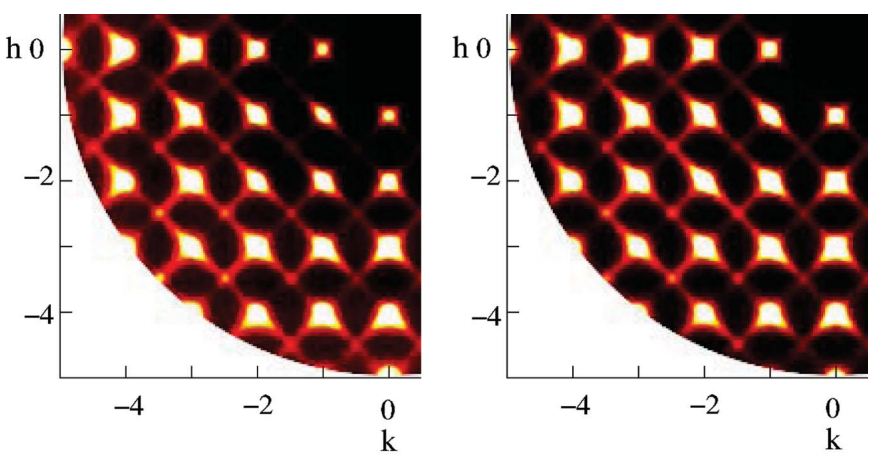

(a) $h k 0$ Neutron

(b) $h k 0 \mathrm{X}$-ray

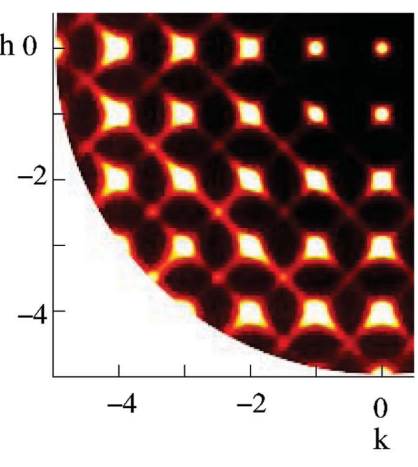

(c) $h k 1$ Neutron

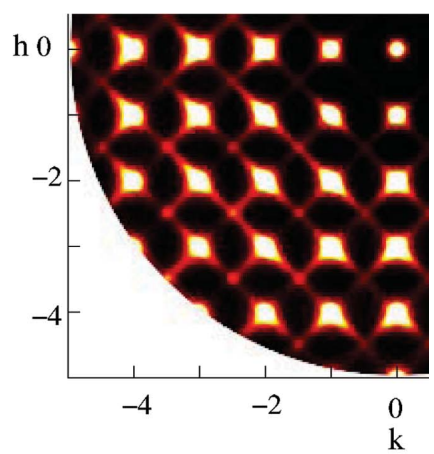

(d) $h k 1$ X-ray

\section{Figure 10}

Diffuse scattering in the $h k 0$ and $h k 1$ planes for both X-ray and neutrons with the BVS MC applied. The changes in the scattering are more subtle than observed in the previous stages. The difference between the before and after BVS MC is shown in Fig. 11, and shows that there is a change in the intensity of alternating diffuse rods of scattering, along with changes to the intensity of the size-effect scattering. 


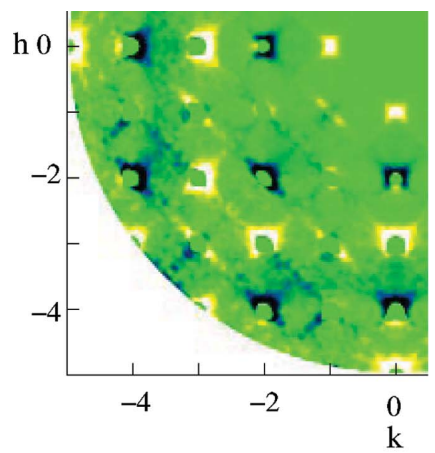

(a) $h k 0$ Neutron

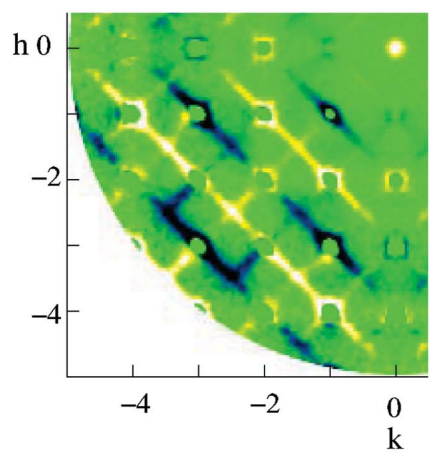

(c) $h k 1$ Neutron

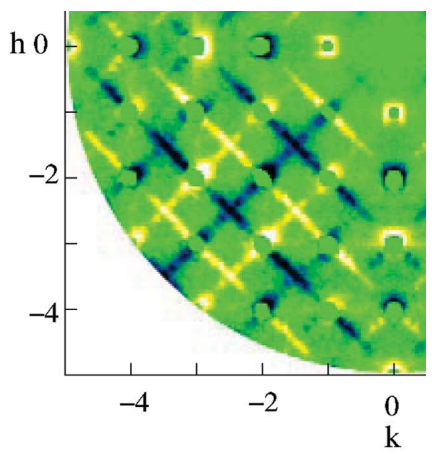

(b) $h k 0 \mathrm{X}$-ray

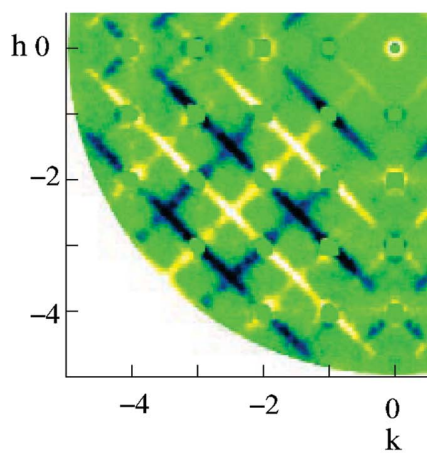

(d) $h k 1$ X-ray

\section{Figure 11}

The change in scattering intensity between the size-effect simulation, Fig. 7 , and the BVS simulation, Fig. 10. The green is zero change in scattering, the white shows increased scattering intensity and the black decreased. The differences for neutron and X-ray are on different scales, as the change in the X-ray scattering is relatively smaller than for neutron.

diffraction pattern which is due to the comparable scattering factor between the $\mathrm{Pb}$ and non- $\mathrm{Pb}$ atoms, unlike $\mathrm{X}$-rays, so the contribution to the scattering of these atoms is more significant.

The changes to the size-effect scattering around the Bragg peaks can be seen to be consistent with what is seen experi-

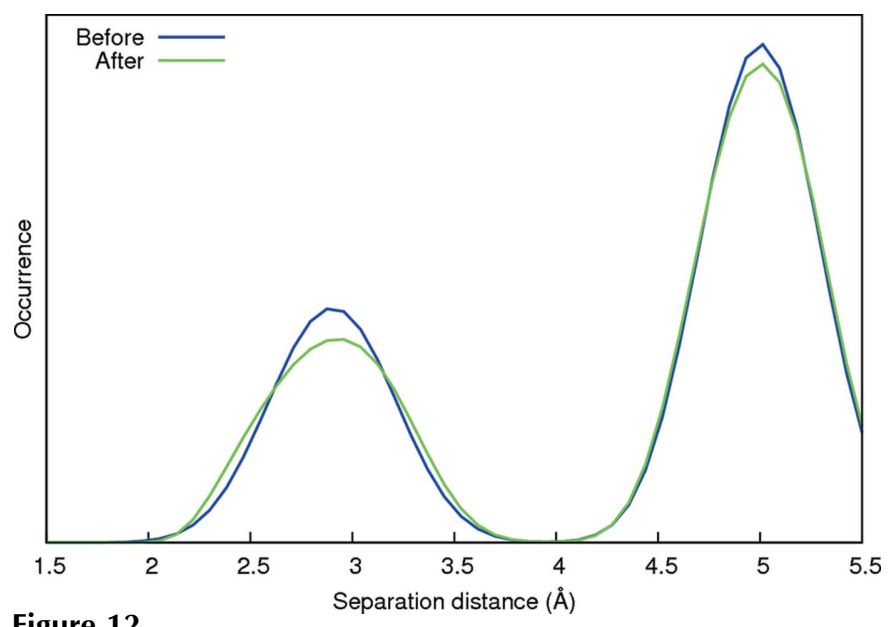

Figure 12

The plot of the distribution of distances between $\mathrm{Pb}$ and $\mathrm{O}$ atoms in the model. The distribution is shown for before and after the BVS MC is applied.

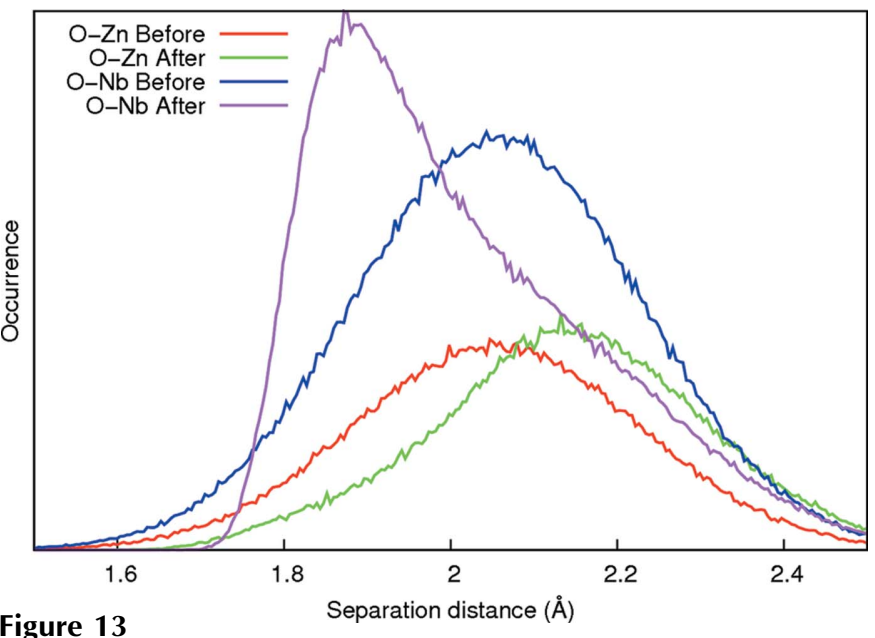

The plot of the distribution of distances between $B$-site and $\mathrm{O}$ atoms in the model. The distribution is shown for before and after the BVS MC is applied.

mentally. There is an increase in the asymmetry of the scattering around the (100) and (300) peaks, while there is a decrease around the (200) and (400) peaks. The other significant difference is the change in intensity of the diffuse rods, with rods alternately increasing and decreasing in intensity, most noticeably in $h k 1$.

The $\mathrm{Pb}-\mathrm{Pb}$ displacement correlations do not change because the $\mathrm{Pb}$-atom positions do not change during this part of the modelling, but a correlation in the displacements of $\mathrm{Pb}$ and $\mathrm{O}$ is observed. The $\mathrm{O}$ atom at the $\left(\frac{1}{2}, \frac{1}{2}, 0\right)$ position is negatively correlated with $\mathrm{Pb}$ in the $x$ and $y$ directions but positively correlated in the $z$ direction. This effect was forced in the earlier model (Welberry et al., 2006) but here arises from the MC energy term based on $\mathrm{Gii}^{2}$.

The plot of the distribution of the $\mathrm{Pb}-\mathrm{O}$ bond lengths is given in Fig. 12 showing the small differences between the distributions before and after the BVS MC simulation. The width of the peaks can be seen to become larger; when moving towards one $\mathrm{Pb}$, an $\mathrm{O}$ is moving away from another, increasing the range of separations.

The plot of the distribution of the separation distances between $\mathrm{O}$ and the different $B$-site atoms, $\mathrm{Zn}$ and $\mathrm{Nb}$, is shown in Fig. 13. It shows that both the atom sites start with the random Gaussian distribution of separation distances. After the BVS MC simulation is run, the distributions change with a shorter separation distance occurring on average between the $\mathrm{O}$ and $\mathrm{Nb}$, than compared to $\mathrm{O}$ and $\mathrm{Zn}$. This is again to do with the requirement for the $B$-site atoms to satisfy their bond valence. The change in the separation distance between the different $\mathrm{O}-(B$-site $)$ atom pair can occur easily when there is alternating ordering of the $B$-site atoms. This is because as the $\mathrm{O}$ is moved closer to a $\mathrm{Nb}$ atom, it is moved further away from the neighbouring $\mathrm{Zn}$ atom.

\subsection{Allowing $\mathrm{Zn}$ and $\mathrm{Nb}$ atoms to swap displacements}

The original model only allowed the displacements, Fig. 10, to be swapped between the $B$ sites if they were of the same 


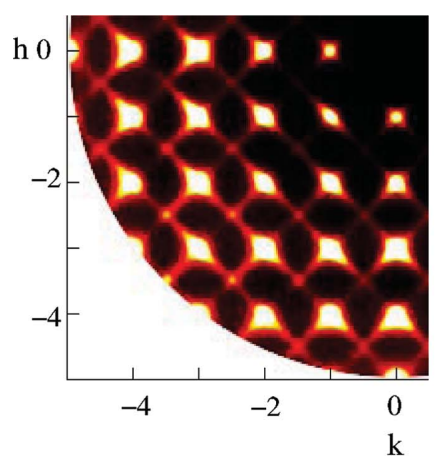

(a) Neutron

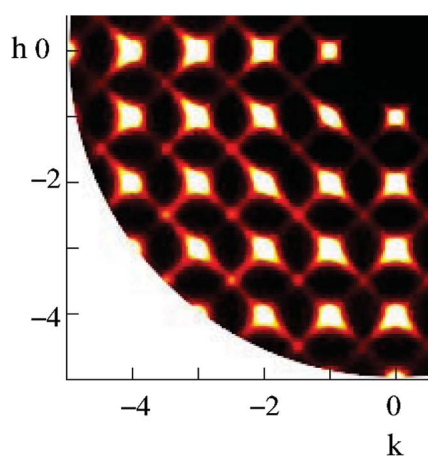

(b) X-ray
Figure 14

The $h k 0$ plane calculated for when the model allows the atom displacements to be swapped between the different $B$-site atoms, $\mathrm{Zn}$ and $\mathrm{Nb}$. The diffuse scattering is almost identical to when the different $B$-site atoms were not allowed to swap atomic displacements.

atom type. By allowing a $\mathrm{Zn}$ to swap its displacement with $\mathrm{Nb}$, the ADPs are less perfectly conserved, but the difference is minor. A benefit is that $\mathrm{Zn}$ and $\mathrm{Nb}$ are not expected to have identical chemical environments, and this allows that to evolve (Fig. 14).

It is found that the overall site thermal parameter is conserved, $U_{\text {iso }}^{(B-\text { site })}=0.015$, but when only looking at a particular atom type it changes, $U_{\text {iso }}^{\mathrm{Zn}}=0.024$ and $U_{\text {iso }}^{\mathrm{Nb}}=0.011$. The $B$ factor of the $\mathrm{Zn}$ site increases while the $\mathrm{Nb}$ decreases as would be expected because $\mathrm{Nb}$ is heavier than $\mathrm{Zn}$. However, there is little impact on the diffuse scattering, so while this may deliver a more realistic model, it is not constrained by the scattering.

\subsection{Changing bond-valence parameters}

It is possible to choose different bond-valence parameters (Mills et al., 2009; Sidey, 2010). In particular, the $\mathrm{Pb}-\mathrm{O}$ bond may be expected to be softer than the $B$-site-O bonds, leading to different values $-R_{\mathrm{O}-\mathrm{Pb}}=1.963$ and $b=0.49$ (Brown, 2009). This changes the balance of energies in the MC simulation. This was found to have a small effect, observable when comparing simulation outputs but too small to allow, for example, refinement of bond-valence parameters against the observed data or determination of which set is 'better'. It also does not change the nature of the model in any significant way.

Having said that, it does raise the possibility of using diffuse scattering to help determine empirical parameters, such as the bond-valence parameters or similar. Since the diffuse scattering is sensitive to the distribution of interatomic distances, in some sense a single diffuse scattering measurement can inform the empirical determination of parameters in potentials in a similar way to using many average structure results to build a histogram (Goossens et al., 2007).

\section{Conclusions}

Bond-valence sums and the resulting measure of structural dissatisfaction, the square of the global instability index, $\mathrm{Gii}^{2}$, can be used as an energy term in Monte Carlo simulation of short-range order in PZN. The approach appears well able to model the behaviour of the non-Pb atoms in the simulation as they adjust to the local structure in the distribution of $\mathrm{Pb}$ ions. The use of the $\mathrm{Gii}^{2}$ as an energy term in the MC simulations of the non- $\mathrm{Pb}$ atoms shows the potential of using the $\mathrm{Gii}^{2}$ for $\mathrm{MC}$ simulations of other types of material.

Such an approach is complementary to others which have been explored elsewhere (Paściak et al., 2012, 2013; Paściak \& Welberry, 2011). These studies made use of the reverse Monte Carlo method to reveal the local structure of PZN. Although both approaches use Monte Carlo simulation, the principles of the two methods are quite different. RMC concentrates on fitting diffuse scattering intensities and in its basic version does not assume any model of the short-range order. This can be advantageous, but also dangerous as careless RMC simulation can lead to a perfect fit, but meaningless results. To avoid this the number of degrees of freedom has to be significantly smaller than the number of data points. Hence, usually rather small systems are studied (for example, $24 \times 24 \times 24$ unit cells). On the other hand, all the atomic correlations have to be represented well, which results in a conflicting demand of the simulation box to be large enough to enclose the biggest correlation length. Simple potentials (like the global instability index) and a 'forward' MC simulation, as used in this paper, allow larger models, avoiding these problems.

As far as the local structure itself is concerned, the RMC results show a very similar structure of correlation to the one obtained here. The main ingredient, anisotropic correlation of $\mathrm{Pb}$ atoms, is qualitatively the same. The correlation coefficient is somewhat smaller in the RMC study, which suggests additional thermal broadening of atomic distributions or differing sensitivities to correlation structure in the two approaches.

The correlations between $\mathrm{Pb}, \mathrm{O}$ and $B$-site cations which were added deterministically in previous models after heuristic interpretation of the diffuse scattering patterns were found to arise 'naturally' through the use of $\mathrm{Gii}^{2}$ as an MC energy. The oxygen environments around $\mathrm{Nb}$ and $\mathrm{Zn}$ were differentiated, as were the ADPs of the two $B$-site cations, all while maintaining the average structure as observed through conventional crystallography.

The model is explicitly in accord with previous structure determinations, because it uses an algorithm in which displacement distributions as determined during these previous studies are maintained because atomic displacement distributions are set up initially based on the values from the literature, and then individual atomic displacements are exchanged subject to the MC energy, preserving the overall distribution.

Regardless of whether the posited short-range-order model is completely correct - and there remains contention over the nature of the displacements, their direction, and even the need for nanodomains themselves - this work shows that the highly structured diffuse scattering, and the local ordering that gives rise to it, can exist in the system without influencing the outcomes of conventional structural studies. This indicates that short-range order may be present, and crucial, and 
unsuspected, in many systems whose structures are thought to be thoroughly determined.

DJG and REW gratefully acknowledge the support of the Australian Institute of Nuclear Science and Engineering. The support of the Australian Research Council and the NCI National Facility at the ANU is also gratefully acknowledged. Use of the Advanced Photon Source was supported by the US Department of Energy, Office of Science, Office of Basic Energy Sciences, under contract No. DE-AC02-06CH11357. Part of this work was sponsored by the Scientific User Facilities Division, Office of Basic Energy Sciences, US Department of Energy.

\section{References}

Adams, St. (2001). Acta Cryst. B57, 278-287.

Bosak, A., Chernyshov, D., Vakhrushev, S. \& Krisch, M. (2012). Acta Cryst. A68, 117-123.

Brese, N. E. \& O'Keeffe, M. (1991). Acta Cryst. B47, 192-197.

Brown, I. D. (2009). Chem. Rev. 109, 6858-6919.

Burkovsky, R. G., Bronwald, Yu. A., Filimonov, A. V., Rudskoy, A. I., Chernyshov, D., Bosak, A., Hlinka, J., Long, X., Ye, Z.-G. \& Vakhrushev, S. B. (2012). Phys. Rev. Lett. 109, 097603.

Burkovsky, R. G., Filimonov, A. V., Rudskoy, A. I., Hirota, K., Matsuura, M. \& Vakhrushev, S. B. (2012). Phys. Rev. B, 85, 094108.

Dmowski, W., Akbas, M. K., Davies, P. K. \& Egami, T. J. (2000). J. Phys. Chem. Solids, 61, 229-237.

Etxebarria, I., Perez-Mato, J. M., García, A., Blaha, P., Schwarz, K. \& Rodriguez-Carvajal, J. (2005). Phys. Rev. B, 72, 174108.

Forrester, J. S., Kisi, E. H., Knight, K. S. \& Howard, C. J. (2006). J. Phys. Condens. Matter, 18, L233.

Goossens, D. J., Welberry, T. R., Heerdegen, A. P. \& Beasley, A. G. (2007). Int. J. Pharm. 343, 59-68.

Harvey, M. A., Baggio, S. \& Baggio, R. (2006). Acta Cryst. B62, 10381042.

Hlinka, J. (2012). J. Adv. Dielectr. 2, 1241006.

Jeong, I. K. \& Lee, J. K. (2006). Appl. Phys. Lett. 88, 262905.
Mills, S. J., Christy, A. G., Chen, E. C. C. \& Raudsepp, M. (2009). Z. Kristallogr. 224, 423-431.

Norberg, S. T., Thomas, P. A. \& Tucker, M. G. (2011). J. Phys. Condens. Matter, 23, 175401.

Norberg, S. T., Tucker, M. G. \& Hull, S. (2009). J. Appl. Cryst. 42, 179184.

Paściak, M., Heerdegen, A. P., Goossens, D. J., Whitfield, R. E., Pietraszko, A. \& Welberry, T. R. (2013). Metall. Mater. Trans. A, 44, 87-93.

Paściak, M. \& Welberry, T. R. (2011). Z. Kristallogr. 226, 113-125.

Paściak, M., Welberry, T. R., Kulda, J., Kempa, M. \& Hlinka, J. (2012). Phys. Rev. B, 85, 224109.

Paściak, M., Wołcyrz, M. \& Pietraszko, A. (2007). Phys. Rev. B, 76, 014117.

Paściak, M., Wołcyrz, M., Pietraszko, A. \& Leoni, S. (2010). Phys. Rev. B, 81, 014107.

Perez-Mato, J. M., Blaha, P., Schwarz, K., Aroyo, M., Orobengoa, D., Etxebarria, I. \& García, A. (2008). Phys. Rev. B, 77, 184104.

Sidey, V. (2010). Acta Cryst. B66, 307-314.

Studer, A. J., Hagen, M. E. \& Noakes, T. J. (2006). Physica B Condens. Matter, 385-386, 1013-1015.

Terado, Y., Kim, S. J., Moriyoshi, C., Kuroiwa, Y., Iwata, M. \& Takata, M. (2006). Jpn. J. Appl. Phys. 45, 7552-7555.

Weber, T. \& Bürgi, H.-B. (2002). Acta Cryst. A58, 526-540.

Weber, T., Estermann, M. A. \& Bürgi, H.-B. (2001). Acta Cryst. B57, 579-590.

Welberry, T. R. (2004). Diffuse X-ray Scattering and Models of Disorder, IUCr Monographs on Crystallography Series. Oxford University Press.

Welberry, T. R. \& Butler, B. D. (1994). J. Appl. Cryst. 27, 205-231.

Welberry, T. R., Goossens, D. J. \& Gutmann, M. J. (2006). Phys. Rev. $B, \mathbf{7 4}, 224108$.

Welberry, T. R., Gutmann, M. J., Woo, H., Goossens, D. J., Xu, G., Stock, C., Chen, W. \& Ye, Z.-G. (2005). J. Appl. Cryst. 38, 639-647.

Whitfield, R. E., Goossens, D. J. \& Studer, A. J. (2012). Metall. Mater. Trans. A, 43, 1429-1433.

Whitfield, R. E., Goossens, D. J., Studer, A. J. \& Forrester, J. S. (2012). Metall. Mater. Trans. A, 43, 1423-1428.

Xu, G., Wen, J., Stock, C. \& Gehring, P. M. (2008). Nat. Mater. 7, 562566. 